\title{
Preferred Biosignals to Predict Migraine Attack
}

\author{
Hanna-Leena Huttunen and Raija Halonen \\ University of Oulu, P.O.Box 4500, 90014 Oulu, Finland \\ \{hanna-leena.huttunen, raija.halonen\}@oulu.fi
}

\begin{abstract}
Migraine is classified to two classes, with aura and without aura, and migraine seizures last usually several hours. The goal of this study was to identify the most important symptoms of migraine to be monitored by wearable sensors to predict the migraine attack. The purpose of wearable sensors is to guide patients to take the migraine medication in time, and to support their own care. Self-measurement is a growing trend worldwide and sensor technology has been used for several years in activity wristbands, smartphones, rings, mobile phones, and mobile applications. The study was conducted as an operational study, randomised for those who had been diagnosed with migraine by a doctor. The study was divided into two parts, at first a questionnaire was sent to 17 people in social media. On the basis of the questionnaire, a qualitative interview was conducted for 12 persons with migraine. Responses to the questionnaire were compared to the results of the interview, and the answers to the research questions were sought. Migraine patients considered important that device reports quality of sleep, pulse, blood pressure, stress levels, sleep apnea, and energy consumption.
\end{abstract}

Keywords: Migraine, Prodromal symptoms, Wearable sensors, Health promotion, Self-measurement.

\section{Introduction}

The purpose of this study was to analyse which biosignals migraine patients would like to measure to help them in managing their migraine. With an intelligent device, the emergence of migraine can be predicted, and patients can be assisted in their own care monitoring. Furthermore, wearable sensors make self-monitoring more efficient and users more aware of their own health (see [1]).

Wearable sensors collect information from users and provide feedback in real time using health applications. Self-measurement technology is about individuals measuring biological, physical, behavioural or environmental information. For several years, selfmeasurement has been a rising global trend utilised for activity wristbands, smartphones, rings, mobile phones and mobile applications. [2,3.]

The aim of the study was to find out the most important biosignals from the view point of patients with migraine that the intelligent device should measure to predict the emergence of migraine attack. In addition, the study investigated the willingness of users to use an electronic migraine diary to support their own care. The main research question was:

The final authenticated version is available online at https://doi.org/10.1007/978-3-319-97931-1_16 
Which are the six most important biosignals a device should measure to predict the incoming migraine attack?

The survey was answered through questionnaires and qualitative interviews. The questionnaires were answered by 17 persons with a migraine diagnosis.

The results of this study show that migraine patients are able to propose the six biosignals that a wearable sensor should measure to detect a migraine attack. With this study, we can define a device that will help migraine patients to detect pre-symptoms and assist them in monitoring their own care and take a medicine in time. Additionally, migraine patients provided researchers with valuable information on the emergence of migraine attacks to develop future devices for migraine treatment.

\section{Related Work}

Migraine can be divided to two classes, with aura and without aura. In migraine with aura, the pain is preceded by visual disturbances, numbness, muscle weakness and difficulty in speech. Migraine without migraine usually begins without the aura symptoms. From neurological diseases, migraine is the most common wide-ranging gene-regulated illness. [4.] Migraine is one of the most expensive diagnosis for European health care as 10 to 15 percent of population suffer from it. Migraine has been associated with other illnesses such as stroke, depression, allergy, and blood pressure. At the European level, migraine costs 111 billion EUR per year. [5,6.] Because of hormonal changes, women suffer from migraine more than men. [7]. Migraine is divided into four stages: pre-symptoms, migraine aura, headache, and post-symptoms. Migraine attacks last for 4-72 hours and change rapidly. Common migraine presymptoms include fatigue, yawning, increased appetite, hunger for sweet, irritability, and feeling cold. The pre-symptoms of migraine with aura lasts for 5-60 minutes, and may include also lightening eyes, smelling sensation, visual field defects, sound sensitivity, and facial acuity. At the end of the aura phase, a pulsating headache begins, and also nausea and vomiting are common. [8.] Migraine can be treated with two types of medication: preventive daily medication and acute medication after detecting presymptoms. The efficacy of medicines depends on which phase of the migraine attack the medication is taken. The best response to treatment with acute medication occurs when the drug is taken before the headache begins. [9.]

Digitalisation is part of future healthcare; the demands of self-care are growing in the world and more effective solutions needs to be developed. [10]. Information and communication technology (ICT) can be used to take the health history of the entire patient into consideration at various stages of treatment. New technology and genetic information enables to design an individual care flow and to prevent the emergence of diseases. Patients can use sensor technology to measure and monitor their own health. Studies show that monitoring of one's own care results better results than using traditional therapy without aid from technology. [7,11.] Evolving technology offers new ways to observe and enhance health and physical fitness. [2,3]. Applications can collect information about user activity and wellbeing using psychical activity trackers, bluetooth-containing heart rate monitors and other sensors. [3]. Sensors used for selfmeasurement have become smaller and can be better used with mobile technologies. [12]. In the United States, about $60 \%$ of adults monitor weight, diet, or fitness, and $33 \%$ 
monitor blood pressure, blood sugar, headaches and sleep. [13]. However, only one in five utilises digital technology to follow these variables, the rest are using older technologies such as pen and paper. [14]. Due to the potential of wearable devices, datagathering internet services and the storage of large data volumes, interest in selfmeasurement and data analysis has increased. [15]. Analysing body and mind functions is no longer exclusive to science, medical researchers and technology specialists, but smart phones make it possible to monitor and store daily life for almost everyone [16].

There are several different devices available on the market, and the interest of the devices to be sold in promoting individuals' own health has received a lot of attention as the new generation of portable devices emerges $[17,18]$. Wearable devices are designed to measure, for example, pulse, daily rest, quality of sleep, activity, stress and mood. Feedback allows users to understand and modify their activities and behaviours and thereby promote their own health. [19.] The structure of wearable devices is generally small and light, and can be attached to, for example, a wrist or incorporated into shoes, clothing or sporting equipment. [20]. Measuring human biosignals from the wrist turned out to be useful in predicting the emergence of diseases. Biosignals provide useful information on physiological changes such as changes in skin temperature, pulse and sleep. The market has advanced sensors that can be used to measure migraine presymptoms. [21,22.] A study of migraine patients revealed their willingness to use sensors to detect pre-symptoms in order to take medication in time. Most of them would like the device to be located in a wrist part of a watch so that migraine alert is easily recognisable. [23.]

Mobile devices, monitors and applications enable healthcare development, as they can be combined with a variety of sensors to collect and evaluate physiological data from the human body. Sensor networks work together via a wireless network. They allow to achieve a wide variety of opportunities to track and understand large-scale, real-life phenomena [7,24.] Mobile devices are used in healthcare and wireless data transfer facilitates efficient data processing [30]. Sensor information is necessary to evaluate the patient's condition, determine the correct diagnosis, and to make treatment decisions and follow-up plans [11].

Increased self-consciousness helps to focus on certain behaviour and can improve well-being [25]. Earlier studies about migraine patients' willingness to wear sensors to detect migraine pre-symptoms and their pre-symptoms revealed that $88.8 \%$ of the respondents wanted a device to detect an upcoming migraine attack. The study also showed that most of the participants wanted the device to be located on the wrist, either in the watch, bracelet, or skin patch [23]. Sleep quality is associated with migraine birth and sleep disorder is a major trigger of migraine, also raising the body's stress hormone [26]. A study of wearable sensor to detect migraine attacks by using the Empatica E4 wristband showed that migraine attacks start at night and migraine patients wake up to headaches. The study also showed that the emergence of migraine attacks is unique and can be detected by sensors. [1.] 


\section{Research Methods}

The study focused on migraine patients by means of qualitative research aimed at understanding a small group in the real world. The main method was qualitative interview, one of the most important tools when collecting qualitative data (see [27]). The study was explorative in nature as very little knowledge was available about the use of body sensors to detect migraines or their pre-symptoms (see [28,29]).

\subsection{Data Collection}

The target group of the study was a random group of migraine sufferers. Collection of the data was carried out in three phases (see Figure 1). In the first phase, people were asked to participate in research through social media. Migraine diagnosis was a criterion for participating. Twenty-two people with migraine came forward and a migraine questionnaire form in October 2016 in cooperation with the migraine Association. There were 17 responses to the questionnaire, which resulted in a qualitative interview for 12 participants. An e-mail survey and a qualitative interview were conducted in the fall of 2017 . Five persons were not interviewed because they did not live in the area. The questions for the interview were compiled on the basis of a literature review. After evaluating the question quality, an invitation letter was sent to the informants.

The email inquiry was open to respondents for two weeks and it was responded only once per person. All questionnaires were correctly filled, so no rejection was made. The questionnaires were analysed and based on analysis the qualitative interview questions were defined. The interviews were conducted with a semi-structured questionnaire with open questions. The study concluded open questions to allow the widest possible picture of the pre-symptoms of migraine and their identification. The interviews were conducted at Oulu during one week, and they lasted about one hour. The interviews were arranged to be pleasant and peaceful to make the setting as natural as possible.

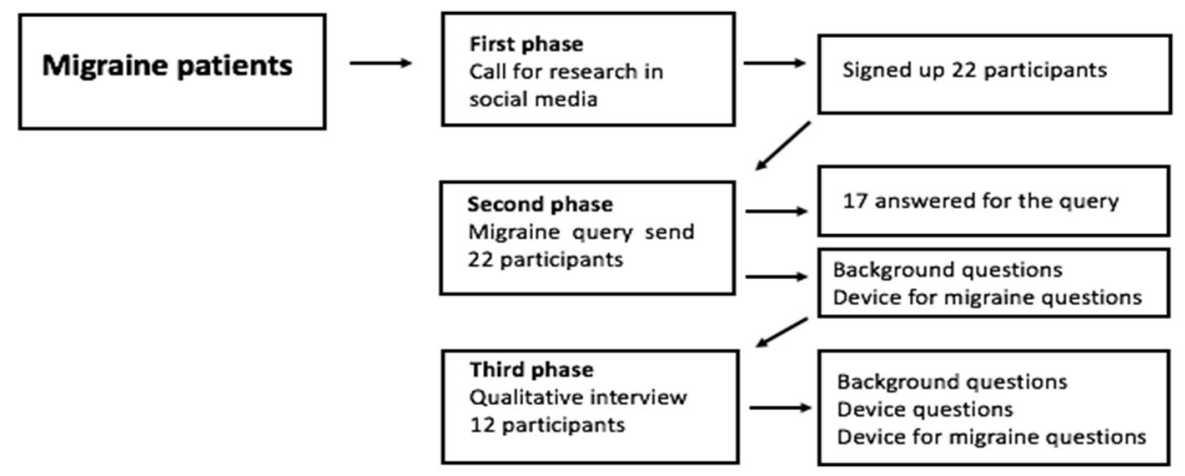

Fig. 1. Qualitative research data collection steps. 


\subsection{Analysis of Data}

The analysis of the questionnaire was divided into two phases. In the first phase, a migraine questionnaire was categorised into two main categories, background information and device issues. The questions of the interview were divided into three main categories, background information, intelligent devices and migraine sensitive sensors. The results of the survey were analysed with Excel tool and cross-table was made. The interview material was read several times to get a full picture and responses were recorded and tabulated. Similarities were sought from the material, and it was organised into categories and named in accordance with the content. The categories were summarised and combined according to the survey and the interview. The background information was classified according to age, sex, hobby, profession, migraine type, pre-symptoms, medication, count of migraine attacks, trigger of migraine attack, time when migraine attacks occur and if relatives suffer from migraine. The data were analysed for typical symptoms associated with migraine and were classified according to the pre-symptoms and the main symptoms.

Questions about the device were classified according to the users' interest, what kind of smart technology users are familiar with, whether the users have wearable devices at home, where the users use wearable devices, what kind of experiences the users have with wearable devices, what motivates the users to wear wearable device, what problems the users have encountered when using wearable devices, and if wearable devices have contributed to the users' health. Questions related to wearable device detecting migraine pre-symptoms were divided according to the pre-symptoms of migraine, whether the users would use a device that identifies the symptoms of a migraine attack, what would the users like the device to measure, whether the users want the device to report directly to the migraine diary, what the users feel about the migraine pre-symptoms device, and how the users feel if the device reports directly to health care information systems.

\section{$4 \quad$ Results}

The material was collected in autumn 2017 via email inquiry and by means of a qualitative interview. There were 17 responses to the questionnaire, but only 12 participants participated in a qualitative interview.

\subsection{Personal Background Questions}

The first phase of the survey was answered by 16 women and one man. Twelve respondents were selected to qualitative interview and all interviewees were women and their age distribution was from 9 to 58 years. Most of them were in the age group of 30-49 years.

Most respondents suffered from migraine with aura, but respondents also had migraine without aura, and one respondent had both. For example Emma, 41 yrs, suffering from migraine with aura described: "Behind the right eye starts a strange feeling, which gradually spreads and intensifies to ache. This is accompanied by sensitivity to odour and light, nausea (no vomiting), intermittent numbness of the head, 
or weakening of thought activity. Duration usually $72 \mathrm{~h}$, usually starting in the morning."

Ann, 34 yrs, suffering from migraine without aura informed: "I feel pulsation in the left eye for multiple days or forehead range. Sometimes also nausea." Jenny, 45 yrs, suffers from migraine both with and without aura and reported about her migraine attacks: "It begins with a powerful headache that medication does not help, but the symptoms worsens. Then there is over sensitivity (tone, light, smells). Nausea, muscle pain, and eye pain."

One respondent had no information on migraine form because the migraine had been diagnosed only recently. Sally, 9 yrs, was not sure if she suffered migraine with aura or not and explained: "First comes stomach pain, fatigue and paleness. After that hard headache. If the medicine is not taken at the right time, I will start to vomit."

Ten respondents were employees, one was unemployed, and one was a schoolchild. All respondents at work worked with computer. All respondents had physical exercise as a hobby.

One questions was to solve if the respondents' relatives had migraine. The respondents had close relatives with diagnosed migraine, and a few respondents had also children with migraine diagnose. Nine mothers and seven sisters suffered from migraine. Four children, two fathers, two brothers and two grandmothers also suffered from migraine. The results proposed that migraine is more common in female relatives. The purpose of the interviews was to find out the typical migraine attacks of the respondents and describe their migraine patterns.

For most respondents, stress, bright lights, excessive clothing, excessive sleeping, blood sugar variations, strong smells, stiffness of the muscles, too heavy exercise, eating chocolate and fatty foods, or menstruation triggered a migraine attack.

The majority of respondents (13) had at least two migraine attacks per month. Two respondents suffered more than five attacks per month, and two respondents suffered 3-4 migraine attacks per month. 14 migraine patients identified migraine symptoms. The most common of them were feeling cold, graving, muscle pain, difficulty finding words and visual disturbances. Three respondents could not classify their presymptoms.

\subsection{Device Questions}

The questions about the device were to determine if the migraine patients used intelligent technology in their everyday life to promote their own health. In the interview, the respondents raised four intelligent devices, but all respondents did not identify smart phones, smartphones, tablets, sports watches and computers as intelligent technologies, so all respondents could not present these four possibilities for the interviewer.

Eleven respondents used a smartphone but one of the respondents couldn't recognise it as smart device. Four respondents recognised that the tablet and computer were intelligent devices that they used in everyday life. Four of the respondents used smartwatch every day in their work, collecting data during sports performance, monitoring their own progress and monitoring sleep quality. The interviewers wanted to know why the respondents had not obtained wearable devices to measure their own health. Most of the respondents mentioned that there is no need to acquire a smart 
device or have not bothered to acquire it. One of the respondents didn't know why he should have a smart device in his own life.

The interviews sought answers to the questions of which of the identified six (6) most important biosignals the device should measure to predict a migraine attack (see Fig. 2). Another question was about pressing a button to synchronise the information with the migraine diary, whether the device should be available through the healthcare provider, and if information should be synced directly to the health care information system.

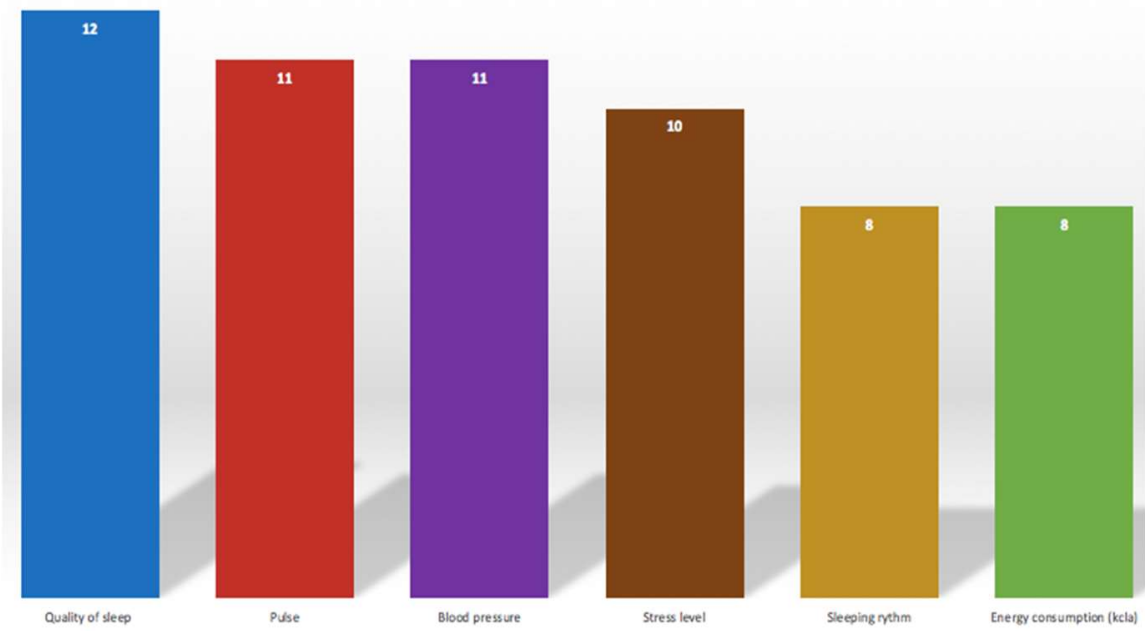

Fig. 2. The six most important biosignals for predicting a migraine attack

Figure 2 lists the desired biosignals revealed by the interviews. Figure 2 shows that all respondents wanted the device to measure sleep of quality (12), next preferred were pulse (11), blood pressure (11), stress level (10), and sleep rhythm and energy consumption were preferred by eight respondents. These six features were raised important for the device to measure to predict the incoming migraine attack.

All 17 respondents who responded to the questionnaire were willing to take the migraine report to the migraine diary. In the interviews, the same question was repeated and all twelve were willing to pass the information to the migraine diary. One respondent said she fills the migraine diary manually and finds it very laborious. The "reset" button would help migraine patients to be able to monitor the frequency of migraine attacks. One respondent explained: "Yes I could press the button. With the migraine diary we could see e.g. the density of migraine attack and think what was triggering the attack." Another told: "The migraine diary would help my life if I could anticipate the attacks and take my medicine in time before the situation gets worse." From the responses, it emerged that the device should be fast and easy to use to maintain interest in the device.

One interviewee described his migraine diary and told how migraine attack had occurred during sports, when the muscles have been tense and when he had been ill. Another reported: "Ten years ago my migraine got chronic and I don't want to get into 
such situation anymore." An anticipating device and migraine diary together help in forecasting pain attacks. One of the patients revealed: 'I'm really afraid of the pain attacks as they take all my ability to act. As a one-parent, one should always be competent to act." The respondents considered it important that the migraine diary is available in a mobile application that can be consulted by a physician / nurse if necessary. They also considered that if they press the acknowledge button, the information goes to the health care information system and is available to the nursing staff and can be visited when needed. One patient confirmed: "All that improves my healthcare I will do. In future, I'd like to know the statistics of how often my migraine knocks and if the attack was prevented by the medicine."

15 respondents considered it important that the device should be available from a health centre, occupational health care, neurologist or other healthcare provider. 12 respondents were willing to buy the device by them self

\section{Discussion}

The aim of the study was to find out if migraine patients would like to monitor their biosignals to reveal pre-symptoms like light sensitive eyes, sound, sensitivity of the smell, facial defect, lack of field of vision, blinking lights in the eyes, or combinations of such symptoms. This study strengthens earlier studies that migraine is a geneticregulated illness and it is inherited from generation to generation. Because of hormonal changes, women suffer from migraine more than men and migraine sickness is located in the 25-44 age group [4,23,1]. In this study most of the respondents were 30-49 years old, and they had been diagnosed with migraine over the years. Respondents' close relatives, such as mothers and daughters, had also been diagnosed with migraine. Of the respondents' mothers, nine had had migraines, seven of daughters had migraines, four children had migraines, and migraine had been diagnosed with two grandmothers. Respondents had migraine with aura and without aura and one of the respondents had no information on the type of migraine. All respondents at work did work with computer. Each respondent had some degree of exercise as a hobby.

Migraine symptoms occur in the majority of patients and symptoms often occur a day before the actual migraine attack. The most common pre-symptoms were fatigue, yawning, increased appetite, hunger for sweet, irritability and feeling cold. [23.] Half of the participants suffered from migraine with aura and they were more likely to detect the oncoming migraine attacks and to medicate themselves in time. Patients with migraine without aura, headaches began unexpectedly but they still suffered from presymptoms, which they could not interpreted as oncoming migraine. Previous study has demonstrated that sleep quality is associated with migraine birth and sleeping disorder is a major migraine trigger and also raises the body's stress hormone [26.] In this study most of the respondents had migraine attack during the night.

Measuring human biosignals on the wrist is proved useful in predicting the emergence of diseases [23]. The six most important biosignals were sleep quality, pulse, blood pressure, stress levels, sleep rhythm, and energy consumption. Biosignals provide useful information on physiological changes that improve the availability of treatment. 
There are various mobile devices on the market, including applications that enable healthcare development, as they can be combined with a variety of sensors to evaluate collected data $[12,24]$. The study shows that migraine patients are willing to support their self care with mobile application, which will automatically record the migraine diary information. The "reset" button would help migraine patients be able to monitor the number and frequency of migraine attacks. Data gathering allows migraine patients to avoid a migraine attack by reacting faster. Feedback allows the user to understand and modify their activities, behaviours and thus promote their own health. [19].

\section{Conclusions}

To conclude, it looks that in the future, more and more health promotion services will become electronic when technology develops at a speed. Technology can help migraine patient in everyday life when pre-symptoms of migraine are easier to identify and treatment can be started faster. Technology helps migraine patients to stay at work for longer, to have less sick leave, and to know their own health care themselves. The sensors used for self-measurement are small and can be used with wearable devices and mobile technology [12]. Earlier research [23] shows that migraine patients want to use a smartwatch or intelligent bracelet that can measure the pre-symptoms of a migraine attack. The current study proposed that migraine patients considered it important that the device measures the quality of sleep, pulse, blood pressure, stress levels, sleep rhythm, and energy consumption. All of the respondents were willing to use a device that reads and syncs the information about migraine attack to migraine diary by a confirm button.

Measuring human biosignals from the wrist is recognised to be useful in predicting emergence of diseases [1] and biosignals provide useful information on physiological changes which can be used to improve the availability of treatment [7,11]. The current study listed sleep quality, pulse, blood pressure, stress levels and sleep rhythms as well as energy consumption as the six most important biosignals of a device to measure.

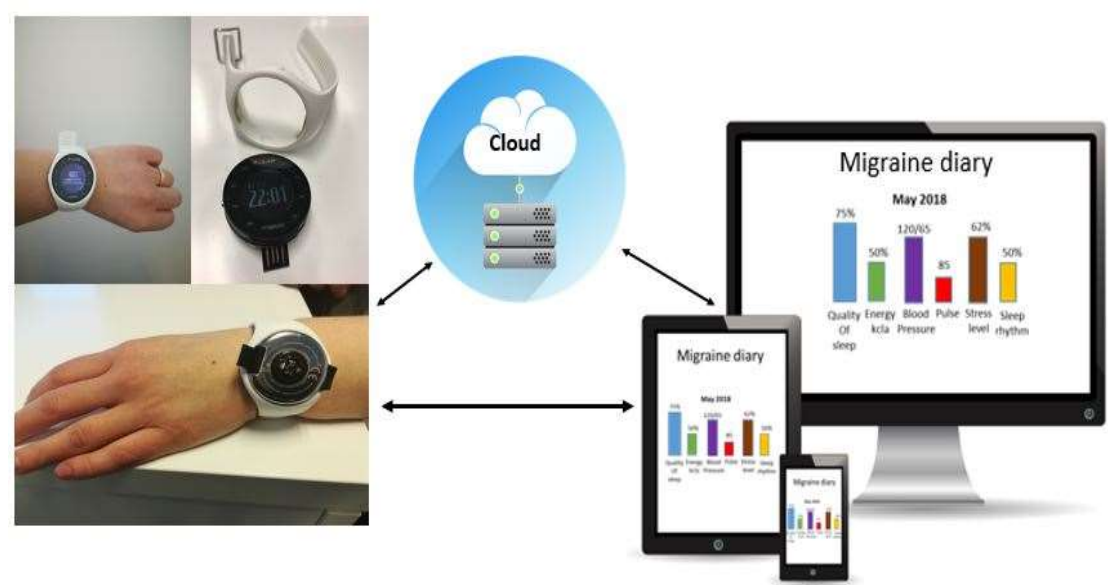

Fig. 3. Architecture for wearables 
The current study proposes that people are willing to use sensor-based wearables that are connected with other systems such as diaries and interaction with other actors. Figure 3 depicts an architectural view for such a system.

As a conclusion, self-measurement is rising worldwide and migraine patients are willing to use smart devices to support their own care. Measuring the pre-symptoms of patients with migraine can promote well-being and illness management.

There are only few studies in the measurement of the pre-symptoms of migraine so far. As studies differ it is difficult to find out how effective a device detecting these six biosignals would be. Further studies are needed to assess the benefits and cost implications of supporting sensors in support and control of migraine in the long term.

\section{References}

1. Koskimäki, H., Mönttinen, H., Siirtola, P., Huttunen, H. L., Halonen, R., \& Röning, J. (2017, September). Early detection of migraine attacks based on wearable sensors: experiences of data collection using Empatica E4. In Proceedings of the 2017 ACM International Joint Conference on Pervasive and Ubiquitous Computing and Proceedings of the 2017 ACM International Symposium on Wearable Computers (pp. 506-511). ACM.

2. Na, H. S., Choi, Y. S., \& Park, T. S. (2009, November). Mobile robot for personal exercise training in ubiquitous healthcare environment. In Proceedings of the 2nd International Conference on Interaction Sciences: Information Technology, Culture and Human (pp. 742744). ACM.

3. Klasnja, Predrag \& Pratt, Wanda: Managing Health with Mobile Technology. interactions, 21(1):66-69, January, 2014, ISSN 1072-5520.

4. Pietrobon, D., \& Striessnig, J. (2003). Neurobiology of migraine. Nature Reviews Neuroscience, 4(5), 386-398.

5. Kaattari, A., Tiirinki, H., Paasivaara, L. Nordstrom, T., \& Taanila, A. (2015). Major user of primary health care services in Northern Finland birth cohort. Social Medication Magazine, 52(3).

6. Kallela, M. (2005). What's new in migraine pathophysiology and genetics. Duodecim,121(6), 665-74.

7. Chan, M., Campo, E., Estève, D., \& Fourniols, JY (2009). Smart homes-current features and future perspectives. Maturitas, 64(2), 90-97.

8. Linde, M, Gustavsson, A., Stovner, LJ, Steiner, TJ, Barre, J., Katsarava, Z., ... \& Ruiz de la Torre, E. (2012). The cost of headache disorders in Europe: The Euro-Light project. European Journal of neurology, 19(5), 703-711.

9. Burstein, R., Collins, B. \& Jakubowski, M. (2004) Defeating migraine pain with triptans: a. race against the development of cutaneous allodynia Annals of Neurology, 55(1), 19-26 Svensson.

10. Olesen J, Gustavsson, A., M, Wittchen, HU, \& Jönsson, B. (2012). The economic cost of brain disorders in Europe.European Journal of Neurology,19(1), 155-162.

11. Ko, J., Lu, C., Srivastava, MB, Stankovic, JA, Terzis, A, \& Welsh, M. (2010). Wireless sensor networks for healthcare. Proceedings of the IEEE, 98(11), 1947-1960.

12. Choe, E., Lee, N., Lee, B., Pratt, W. \& Kientz, J. (2014). Understanding quantified-selfers' practices in collecting and exploring personal data. In Proceedings of the 32nd annual ACM conference on Human factors in computing systems (CHI '14). 1143-1152. 
13.Swan, M. (2013). The quantified self: Fundamental disruption in big data science and biological discovery. Big Data, 1(2), 85-99.

14. Lupton, D. (2016). The diverse domains of quantified selves: self-tracking modes and dataveillance. Economy and Society, Volume 25, Issue 1. 101- 1229.

15. Rettberg, J. W. (2016). Seeing ourselves through technology: How we use selfies, blogs and wearable devices to see and shape ourselves.

16. Pantzar, M. \& Ruckenstein, M. (2014). The heart of everyday analytics: emotional, material and practical extensions in self-tracking market, Consumption Markets \& Culture, 18:1, 92-109.

17. Fritz, T., Huang, E., M. Murphy, G. C. \& Zimmermann, T. (2014). Persuasive technology in the real world: a study of long-term use of activity sensing devices for fitness. Proceedings of the SIGCHI Conference on Human Factors in Computing Systems 487-496.

18. Yang, H., Yu, J., Zo, H. \& Choi, M. (2016). User acceptance of wearable devices: An extended perspective of perceived value. Telematics and Informatics, 33(2), 256-269.

19.Crawford, K., Lingel, J. \& Karppi, T. (2015). Our metrics, ourselves: A hundred years of self-tracking from the weight scale to the wrist wearable device. European Journal of Cultural Studies, Vol 18, Issue 4-5, (s. 479 - 496).

20. Parviainen, J. (2016). Quantified Bodies in the Checking Loop: Analyzing the Choreographies of Biomonitoring and Generating Big Data. Human Technology, 12 (1), 5673.

21. Pagán, J., Orbe, D., Irene, M., Gago, A., Sobrado, M., Risco-Martín, J. L., ... \& Ayala, J. L. (2015). Robust and accurate modeling approaches for migraine per-patient prediction from ambulatory data. Sensors, 15(7), 15419-15442.

22. Pagán, J., Risco-Martín, J. L., Moya, J. M., \& Ayala, J. L. (2016, July). Grammatical evolutionary techniques for prompt migraine prediction. In Proceedings of the Genetic and Evolutionary Computation Conference 2016 (pp. 973-980). ACM.

23. Huttunen, H. L., Halonen, R., \& Koskimäki, H. (2017, September). Exploring use of wearable sensors to identify early symptoms of migraine attack. In Proceedings of the 2017 ACM International Joint Conference on Pervasive and Ubiquitous Computing and Proceedings of the 2017 ACM International Symposium on Wearable Computers (pp. 500505). ACM.

24. Krishnamachari, B. (2005). Networking wireless sensors. Cambridge University Press.

25. Bentley, F., Tollmar, K., Stephenson, P., Levy, L., Jones, B., Robertson, S., Price, E., Catrambone, R., \& Wilson, J. (2013). Health mashups: Presenting statistical patterns between wellbeing data and context in natural language to promote behavior change. ACM Trans. Comput.-Hum. Interact. 20, 5, Article 30 (November 2013), 27 pages. DOI:http://dx.doi.org/10.1145/2503823.

26. Mönttinen, H., Koskimäki, H., Siirtola, P., \& Röning, J. (2017). Electrodermal activity asymmetry in sleep - a case study for migraine detection. In EMBEC \& NBC 2017 (pp. 835838). Springer, Singapore.

27. Myers, M. D., \& Newman, M. (2007). The qualitative interview in IS research: Examining the craft. Information and organization, 17(1), 2-26.

28. Gable, G. G. (1994). Integrating case study and survey research methods: an example in information systems. European journal of information systems, 3(2), 112-126.

29. Baskerville, R. L. (1999). Investigating information systems with action research. Communications of the AIS, 2(3es), 4.

30. Thiyagaraja, S. \& Dantu, R. (2013), Finger Blood Flow Monitoring Using Smart Phones. Book. Proceedings of the 8th International Conference on Body Area Networks, BodyNets '13, pages 237-239, ICST, Brussels, Belgium, Belgium. ISBN 978-1-936968-89-3. 